\title{
Eventos adversos en niños hospitalizados: un desafío para el Cuidado de Enfermería
}

\author{
Adverse events in hospitalized children: a challenge for Nursing Care \\ Eventos adversos em crianças hospitalizadas: um desafio para o Cuidado de Enfermagem \\ Cielo Rebeca Martínez-Reyes* \\ Fayzuri Álvarez-Reyes** \\ Cesar Augusto Garzón-Martínez ${ }^{* * *}$ \\ Isabel Cristina Rave-Henao ${ }^{* * * *}$
}

\section{Resumen}

Introducción: La enfermería, profesión con raíces humanistas, preocupada por las personas que confían en su cuidado, tiene como desafío garantizar la seguridad de los pacientes, incluidos los niños. Metodología: Se realizó una revisión de artículos científicos publicados entre 2010 y 2017, en las bases de datos NCBI, Lilacs, Redalyc, Scielo, Elsevier, Medigraphic, Google Académico y Science Direct; escritos en español, inglés y portugués que cumplían con criterios de pertinencia metodológica y temática. Las palabras clave empleadas fueron: eventos adversos, enfermería pediátrica y seguridad del paciente. Los artículos fueron evaluados con las escalas Prisma, Strobe y Coreq. Resultados: Se seleccionaron 14 artículos que analizaban eventos adversos propios del cuidado de enfermería en niños hospitalizados relacionados con: medicamentos, infecciones, flebitis y úlceras por presión, en los cuales se concluía que factores propios del infante, los dispositivos utilizados, el ambiente institucional y el actuar del personal, pueden incidir en la ocurrencia de errores que deterioran la salud del niño. Conclusiones: Apropiarse de una cultura de seguridad que incluya la adherencia a protocolos y la reafirmación de conocimientos sobre prácticas seguras, basadas en la mejor evidencia científica, son estrategias infalibles en la mitigación y prevención de los eventos adversos en el cuidado de enfermería.

Palabras clave: Cuidado de enfermería, eventos adversos, niños hospitalizados, seguridad del paciente.

\section{Abstract}

Introduction: Nursing, a profession with humanist roots, concerns about people who trust in its care, and has the challenge of guaranteeing the patients safety, including children. Methodology: A review was performed on scientific articles published between 2010 and 2017, from the data bases NCBI, Lilacs, Redalyc, Scielo, Elsevier,

\section{Autor de correspondencia}

* $\square *$ Enfermera, Magister en Gestión de la Calidad de los Servicios de Salud. Docente del Programa de Enfermería. Corporación Universitaria Adventista. E-mail: rebeca@ unac.edu.co. Orcid iD https://orcid.org/00000003-1545-5094. Medellín, Colombia.

** Estudiante IX Semestre de Enfermería. Corporación Universitaria Adventista. Email: fayalvarez@unac.edu.co. Orcid: (ID https:/orcid.org/0000-0002-1697-0218. Medellín, Colombia.

*** Estudiante IX Semestre de Enfermería. Corporación Universitaria Adventista. Email: cgarzon@unac.edu.co. Orcid: https://orcid.org/0000-0003-3149-5264. Medellín, Colombia.

**** Estudiante IX Semestre de Enfermería. Corporación Universitaria Adventista. Email: isaravehenao@gmail.com. Orcid: (DD https://orcid.org/0000-0001-7575-302X. Medellin, Colombia.

Este es un artículo bajo la licencia CC BY

(https://creativecommons.org/ licenses/by/4.0/) (c) (1) 
ISSN-PRINT

1794-9831

E-ISSN 2322-7028

Vol. 16 No. 1

Ene - Abr 2019

Cúcuta, Colombia

Medigraphic, Google Scholar and Science Direct; written in Spanish, English and Portuguese and met the pertinent methodological and subject criteria. The keywords employed were: adverse events, pediatric nursing and patient safety. The articles were evaluated with the PRISMA, STROBE and COREQ guidelines. Results: 14 articles were selected, these analyzed adverse events proper of nursing care in hospitalized children related to: medication, infections, phlebitis and pressure ulcers, concluding that factors specific to the infant, used devices, institutional environment and personnel performance incites the occurrence of errors that deteriorate the health of the child. Conclusions: Appropriating a safety culture that includes the adherence to protocols and the reaffirmation of knowledge about safe practices based on the best scientific evidence, are infallible strategies in the mitigation and prevention of adverse events in nursing care.

Keywords: Nursing care, adverse events, hospitalized children, patient safety.

\section{Resumo}

Introdução: A enfermagem, profissão com raízes humanistas, preocupada pelas pessoas que confiam em seu cuidado, tem como desafio garantir a segurança dos pacientes, incluídas as crianças. Metodologia: Realizou-se uma revisão de artigos científicos publicados entre 2010 e 2017, nas bases de dados NCBI, Lilacs, Redalyc, Scielo, Elsevier, Medigraphic, Google Acadêmico e Science Direct; escrito em espanhol, inglês e português que preencheram critérios de relevância metodológica e temática. As palavras chave empregadas foram: eventos adversos, enfermagem pediátrica e segurança do paciente. Os artigos foram avaliados com as escalas Prisma, Strobe e Coreq. Resultados: Foram selecionados 14 artigos que analisavam eventos adversos próprios do cuidado de enfermagem em crianças hospitalizadas relacionados com: medicações, infecções, flebites e úlceras por pressão, nos quais se concluía que fatores próprios do bebê, os dispositivos utilizados, o ambiente institucional, o agir do pessoal, podem incidir na ocorrência de erros que deterioram a saúde da criança. Conclusões: Criar uma cultura de segurança que inclua a aderência a protocolos e a reafirmação de conhecimentos sobre práticas seguras, baseadas na melhor evidência científica, são estratégias infalíveis na mitigação e prevenção dos eventos adversos no cuidado de enfermagem.

Palavras-chave: Cuidado de enfermagem, eventos adversos, crianças hospitalizadas, segurança do paciente.

\section{Introducción}

La enfermería es una profesión con profundas raíces humanistas, genuinamente preocupada por las personas que confían en su cuidado, con especial énfasis en quienes experimentan una situación de enfermedad y sufrimiento (1). En ese sentido, como disciplina profesional, tiene por naturaleza y paradigma el cuidado, brindando asistencia y soporte, con el fin de aliviar las condiciones de salud en una población que atraviesa por una situación de vulnerabilidad (2).

En consecuencia, tanto el cuidado de enfermería, como la atención en salud en general, deben ser brindados con calidad, lo que implica la seguridad del paciente, definida por el Ministerio de la Protección Social de Colombia "como el conjunto de elementos estructurales, procesos, instrumentos y metodologías basadas en evidencias científicamente probadas que propenden por minimizar el riesgo de sufrir un evento adverso (EA) en el proceso de atención de salud o de mitigar sus consecuencias" (3).

Por ello, garantizar la seguridad de todas las personas que utilizan los servicios de salud es uno de los más importantes desafíos que enfrentan los países en la actualidad (4-8). La seguridad de los pacientes es una prioridad de salud pública expresada en las políticas de organizaciones nacionales e internacionales que velan por la calidad de los servicios $(3,5,9)$. La alta prevalencia de EA que se reporta en las investigaciones, ha llevado a que se catalogue como una prioridad global. Tan sólo en Estados Unidos, el Instituto de Medicina indica que alrededor de 100.000 pacientes morían cada año, no como producto de sus enfermedades, sino como resultado de errores en la atención brindada en los servicios de salud (10). Desde entonces, cada vez son mayores los esfuerzos que se realizan para mejorar la seguridad de la atención, prevenir errores que puedan generar invalidez o muerte de los 
pacientes $(3,5,11-12)$, y que también generan efectos económicos y legales contra los profesionales y las instituciones (4-5,13-15).

Aunque la seguridad es responsabilidad de todos los involucrados en la prestación de los servicios de salud, el personal de enfermería permanece todo el tiempo al cuidado de los pacientes, por esa razón, tiene mayor contacto directo con él, y dada la complejidad de la práctica clínica, su desempeño, influye de manera directa en la ausencia o presencia de eventos adversos (3,15-17), los cuales son definidos como un resultado desfavorable por acción o por omisión de la atención en salud que se produce como consecuencia de situaciones que pueden evitarse con el ajuste de normas y procedimientos $(3,5-6,15,17-20)$.

Se debe precisar, que estos sucesos, están relacionados con múltiples factores, que van desde la alta complejidad de la atención, el cambio constante de las condiciones clínicas de los pacientes, el grado de dificultad de cada procedimiento y por supuesto, los factores humanos $(15,21-23)$.

A propósito, el Estudio IBEAS, realizado en Colombia, México, Costa Rica, Perú y Argentina, puso en evidencia que "los EA detectados estaban relacionados con los cuidados en un $13,27 \%$, con el uso de la medicación en un $8,23 \%$, con la infección nosocomial en un $37,14 \%$, con algún procedimiento en un $28,69 \%$ y con el diagnóstico en un $6,15 \%$. Los cinco EA más frecuentes fueron las neumonías $(9,4 \%)$, las infecciones de herida quirúrgica $(8,2 \%)$, úlceras por presión $(7,2 \%)$, otras complicaciones relacionadas con intervención quirúrgica o procedimiento $(6,4 \%)$ y sepsis o bacteriemia (5\%), acumulando un total del $36,2 \%$ de los EA identificados; en particular, los EA en pacientes pediátricos en este estudio tuvieron una prevalencia del 12,7\% (18).

Como puede observarse, tales eventos están íntimamente relacionados con la labor que día a día realiza el equipo de enfermería, situación que es similar a la de otras investigaciones, que develan que las infecciones hospitalarias, seguida de fallas en administración de los medicamentos, flebitis, úlceras por presión y caídas, son eventos adversos frecuentes en el cuidado de enfermería brindado a los pacientes en general $(5,11,24-29)$.

Si bien es cierto, que todos los pacientes están en condiciones de vulnerabilidad ante el error humano de quienes los cuidan, los niños lo son aún más $(11,30)$. Asimismo, la literatura reporta que en los niños, existe mayor riesgo de infecciones, flebitis, úlceras por presión y errores con la administración de medicamentos, por lo cual resulta favorecedor abordar no solo su caracterización, sino además, analizar factores de riesgo y estrategias para minimizarlos, con el propósito de acercar tales alternativas a nuestro contexto, proporcionando a los profesionales estrategias basadas en la evidencia de que se pueden implementar en el quehacer del cuidado de enfermería. Por ello se planteó como objetivo identificar los EA comunes en pediatría derivados del cuidado de enfermería, sus factores de riesgo y las medidas que disminuyan su ocurrencia.

\section{Metodología}

Se llevó a cabo una revisión de la literatura durante el segundo semestre de 2017, teniendo en cuenta que los artículos seleccionados cumplieran con los siguientes criterios de elegibilidad:

- Artículos de estudios cuya muestra estuviera conformada por pacientes pediátricos en condiciones de hospitalización y que presentaron EA con relación al cuidado de enfermería; publicados en español, inglés y portugués, entre los años 2010 y 2017 en revistas indexadas con categoría A, B y $\mathrm{C}$, según Publindex.

- Artículos que de acuerdo con su naturaleza (observacionales, experimentales y cualitativos), cumplieran con los criterios de evaluación de las listas PRISMA (31-32), STROBE (32-34) y COREQ (35).

En cuanto a los términos de búsqueda, fueron empleadas palabras clave y términos $\mathrm{MeSH}$ : eventos adversos, enfermería pediátrica y seguridad del paciente, y sus equivalentes en inglés y portugués, consultados en bases de datos electrónicas de acceso libre como NCBI, Lilacs, Redalyc, Scielo, Medigraphic, Google Académico y Science Direct.

Tras esta búsqueda se hallaron 72 artículos originales, de revisión y de reflexión publicados entre los años 2010 y 2017 relacionados con el tópico de interés. Luego, se verificó que el título y resumen, metodología y resultados de cada artículo, estuviesen relacionados con del tema en cuestión. 
ISSN-PRINT

1794-9831

E-ISSN 2322-7028

Vol. 16 No. 1

Ene - Abr 2019

Cúcuta, Colombia
De esta forma se preseleccionaron 18 artículos que fueron estudiados utilizando una matriz de análisis, teniendo en cuenta los siguientes datos: nombre del estudio, base de datos de origen, clasificación de la revista según Publindex-Colciencias, formación académica de los autores, fecha de la investigación, país en que se realizó el estudio, tipo de estudio, tema objeto de investigación, muestra, descripción de resultados y fecha de publicación.

Con posterioridad, estos documentos fueron evaluados mediante la utilización de listas de chequeo, según el tipo de artículo, siguiendo los siguientes patrones: los cuatro documentos de revisión se analizaron a la luz de los criterios Preferred Reporting Items for Systematic Reviews and Meta-Analyses - PRISMA (31-32); los 13 originales que plasmaban estudios observacionales, fueron sometidos a las pautas de Strengthening the Reporting of Observational studies in Epidemiology-STROBE (32-34); y un artículo que trataba de un estudio cualitativo, fue examinado mediante Consolidated Criteria for Reporting Qualitative Research - COREQ. (35)

Lo anterior permitió, que en los artículos de revisión y de estudios observacionales se analizara con claridad, coherencia y pertinencia de lo sustentado en cuanto a: su estructura en la exposición del título, resumen, introducción, objetivos, resultados y discusión; también se examinó la metodología, verificando el diseño, muestra, variables estudiadas y manejo estadístico. En el caso del artículo con enfoque cualitativo, se tuvieron en cuenta: la comunicación lógica del método empleado, contexto del estudio, resultados, análisis e interpretación.

Finalmente, en este último filtro se excluyeron cuatro, quedando depurados 14 artículos que hacen parte de la revisión.

De igual manera, se aclara que con el propósito de minimizar los sesgos en la investigación en donde se tiende a incluir estudios con resultados positivos frente al objeto de estudio, se delimitaron claramente los criterios de inclusión y exclusión. Para evitar este sesgo en la publicación se realizó la búsqueda en varias bases de datos indexadas empleando términos $\mathrm{MeSH}$ y palabras clave.

Con respecto a las consideraciones éticas, según lo dispuesto en la Resolución 008430 de 1993 mediante la cual se dictan las normas técnicas y científicas para la investigación en Colombia, esta revisión se considera sin riesgo, porque no se requirió intervención a seres humanos (36).

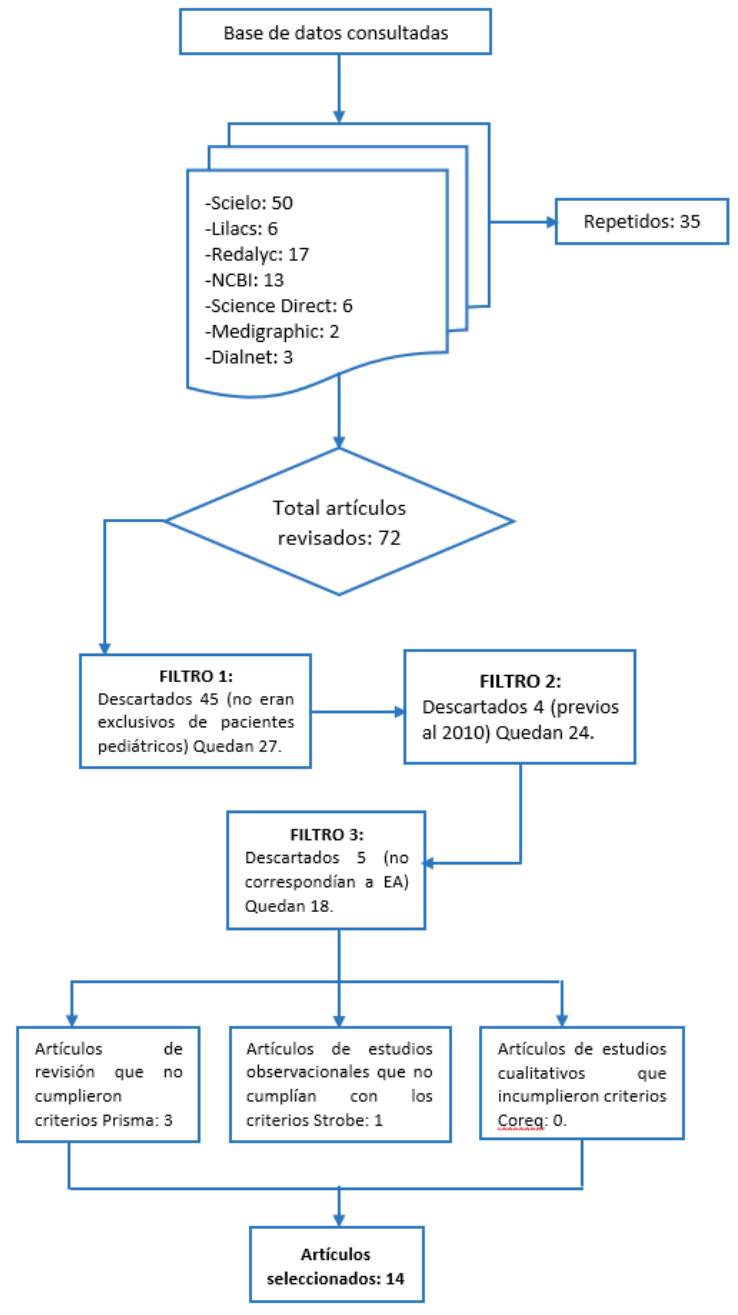

Figura 1. Proceso de selección de Artículos Fuente: Proceso de búsqueda hecho por los autores

\section{Análisis}

De los 14 artículos seleccionados, tres son de Brasil, dos de México, dos de España y dos de Chile. De Argentina, Uruguay, Estados Unidos, Venezuela y Turquía se eligió un artículo respectivamente. En lo que corresponde al idioma en el que estaban escritos: $3(21 \%)$ estaban en inglés, uno (7.1\%) en portugués y el resto español 71.4\%). Las bases de datos con más artículos escogidos fueron: Scielo (42.8\%), seguida de NCBI (28.5\%), Redalyc (21.4\%) y Medigraphic (9.1\%). Tres de los artículos estaban publicados en revistas $\mathrm{A} 1$ e igual número en las de categoría $\mathrm{B}$, dos en $\mathrm{A} 2$ y seis en C. 13 artículos tienen un enfoque 
cuantitativo, en su mayoría fueron descriptivos y de cohortes, seguidos de un artículo de revisión y un artículo cualitativo fenomenológico.

Dentro del tema de eventos adversos en el cuidado de enfermería, los artículos seleccionados, detallan aspectos relacionados con los factores asociados a su ocurrencia y tratan de manera específica de situaciones como flebitis, úlceras por presión, Infecciones Asociadas al Cuidado de la Salud (IACS) y Eventos Adversos por Medicamentos (EAM).

\section{Eventos adversos en pediatría y sus factores rela- cionados}

Un evento adverso es una situación indeseable, que redunda de manera negativa en la condición del paciente, ocurre durante la atención de salud de manera no intencional (17).

La seguridad del paciente y la reducción de los EA, son hoy en día temas de gran relevancia para todos los países. En el ámbito de la pediatría, un estudio realizado en Argentina sobre su prevalencia, se reportó que, de 484 historias clínicas revisadas, se encontraron 146 EA en 82 hospitalizaciones (16,9\%), es decir, un promedio de tres por cada 10 hospitalizaciones, de las cuales el $90 \%$ pudieron ser evitadas (17). Estos hallazgos están vinculados con los reportes realizados por Requena (37)the Asturias Study on hospitalisation -related adverse events (EAPAS en España, en donde los EA más frecuentes fueron: los relacionados con la medicación (38\%) y con los cuidados $(17,2 \%)$, seguidos pord infección asociada al cuidado $(13,8 \%)$ y con el diagnóstico (10,3\%). Respecto a la evitabilidad según el tipo de EA, los menos evitables fueron los relacionados con los cuidados.

Los artículos coinciden en informar que la etiología de los EA tiene una naturaleza multicausal, dentro de los que se pueden resaltar según Riquelme et al., el estrés y la sobrecarga laboral en el personal de enfermería debido a jornadas laborales extensas y sobrepoblación de pacientes, $(17,38)$ a lo que se suma la falta de destreza y la habilidad técnica para enfrentar estas situaciones. Se ha observado que la jornada de la mañana es donde más EA ocurren (39), lo cual podría estar asociado al mayor número de intervenciones que se realizan durante este tiempo. Por ello, Wegner et al., insisten en la supervisión permanente y en mejorar la efectividad de la comunicación, como parte de la adopción de una cultura de seguridad del paciente, factores indispensables en la reducción y prevención de los EA (40).

\section{Eventos adversos derivados del uso de medica- mentos}

Un evento adverso por medicamentos (EAM), se define como un incidente evitable, secundario a la utilización inapropiada de fármacos que causan o pueden causar daño al paciente durante la atención en salud, lo cual debe diferenciarse de una reacción adversa, que es siempre inevitable, como todo efecto perjudicial y no deseado que se presenta después de la administración de un medicamento, a las dosis normalmente utilizadas en el hombre $(38,41)$.

Gutiérrez et al., sostienen que la prevalencia de estos errores en pacientes adultos y pediátricos es diferente, con una incidencia tres veces mayor en niños hospitalizados (38). Su etiología es de origen multifactorial, sin embargo, existen condiciones fisiológicas que hacen a los niños especialmente vulnerables lo que implica que el personal de enfermería extreme la vigilancia para conseguir los mejores resultados terapéuticos al menor riesgo. De hecho, se ha comprobado que la farmacocinética y la farmacodinamia en la población infantil son diferentes a las de los adultos (42).

En cuanto a la absorción, Tayman et al., informan que ésta varía según la vía de administración. Por ejemplo, por vía oral al llegar al estómago, el medicamento es afectado por el $\mathrm{pH}$ y cantidad de jugo gástrico, los cuales dependen de la edad y se acercan a valores de un adulto solo hasta el segundo año de edad. Dado que la alimentación extrauterina, incluida la lactancia materna aumenta la acidez estomacal, los fármacos ácidos débiles se absorban más rápidamente (42).

Entre tanto, por vía intramuscular, la absorción se hace más lenta dado que hay poco tejido muscular con poca irrigación; mientras que la vía intrapulmonar local es más efectiva que la sistémica porque el recién nacido tiene inmadurez alveolar y por tanto una capacidad reducida para el intercambio gaseoso (42). De la distribución y metabolismo, es importante resaltar que la albúmina y las glicoproteínas a las que se unen los fármacos están disminuidas en recién nacidos y niños pequeños, lo que aumenta la cantidad de compuesto activo libre y con ello su disponibilidad y distribución en los tejidos, produce efectos adversos (42). 
ISSN-PRINT

1794-9831

E-ISSN 2322-7028

Vol. 16 No. 1

Ene - Abr 2019

Cúcuta, Colombia
Finalmente, la inmadurez renal, dada por la escasa filtración glomerular, el flujo sanguíneo, y la secreción tubular afectan la eliminación de los fármacos. Estas funciones solo se completan de los siete a 12 meses de edad (42).

Con relación con la farmacodinamia, es ampliamente conocido que los medicamentos pueden alterar, detener o retrasar el crecimiento en la infancia, es decir, afectan los órganos aún en desarrollo. Además, los rápidos cambios de masa, forma y composición corporal son un desafío para conseguir la dosificación adecuada (42).

Haciendo referencia a los EAM, Esqué et al., en su estudio detectaron 511 en una unidad neonatal, dentro de los que se destacan: fármacos $65,4 \%$, alimentación parenteral $9.2 \%$, líquidos endovenosos $8.6 \%$, óxido nitroso $0.4 \%$ y oxígeno $0.8 \%$. Se determinó una incidencia de 32,2 por 1.000 días de hospitalización o 0,2 por paciente, de los cuales el 0,22 por 1.000 días tuvieron repercusiones graves; el 39,5\% fueron errores de prescripción y el $68,1 \%$ de administración (41).

Los errores más frecuentes reportados fueron los relacionados con: terapia de antibióticos, sedación, analgesia, diuréticos, colirios, insulinas e inotrópicos, fallando en el intervalo de administración en un $65.4 \%$, dosis incorrecta el 33,1\%. Fue interceptado el 17\%. La mayoría de los EAM sucedieron en la unidad de críticos (72\%). El grupo profesional que tuvo menos errores fue el de 5 a 10 años de antigüedad (21,6\%); y el personal de enfermería el que más los notificó $(69,5 \%)(41)$.

Asimismo, en otra investigación se revisaron 136 historias clínicas durante 24 horas, en las que había 448 transcripciones de enfermería, de las cuales el $78 \%$ tenían errores: como ausencia de fecha, hora y firma, medicamento, dosis, frecuencia y vía equivocados. Los medicamentos involucrados en estos eventos fueron: soluciones intravenosas, antibióticos, broncodilatadores, oxígeno, analgésicos y antipiréticos (38).

Los estudios analizados coinciden en reconocer la necesidad del conocimiento de la farmacocinética y farmacodinamia de los niños para disminuir el riesgo de error. Igualmente exhortan a que el equipo de salud reflexione sobre la responsabilidad que implica la prescripción, transcripción y administración de medicamentos de igual manera se resalta que el grupo de médicos, enfermería y farmacia hospitalaria supervisen mejor esta tarea y analicen los errores cuando se producen o cuando "casi" se producen. Se debe crear un ambiente adecuado para desarrollar programas de "medicación segura" para los pacientes y garantizar así la seguridad de la población infantil $(38,41-42)$.

\section{Infecciones asociadas al cuidado de la salud (IACS)}

Son las contraídas por un paciente durante su tratamiento en un hospital u otro centro sanitario, no presente ni incubándose en el momento de su ingreso. Incluso, pueden aparecer después del alta (43) y en niños afectan principalmente el tracto respiratorio, gastrointestinal, de sistema nervioso central, urinario o vascular (44-45).

En el contexto de la pediatría, estas infecciones constituyen un problema de salud pública, teniendo en cuenta que alargan la estancia hospitalaria; también aumentan: la discapacidad a largo plazo, la resistencia de los microorganismos a los antibióticos y el riesgo de morbimortalidad de los pacientes, constituyéndose en un indicador de calidad del centro prestador de servicios de salud.

Los estudios de Flores et al. (44) y López et al. (45), encontraron que alrededor de un $12 \%$ de los pacientes se infectaron, afección siendo más frecuente en los varones. También hallaron que los sistemas afectados son las vías respiratorias bajas, gastrointestinal con diarreas agudas e infecciones de catéteres vasculares de vía urinaria (44) y de derivación ventriculoperitoneal (45). Los agentes implicados en tales infecciones fueron: Rotavirus, virus parainfluenza 3(44), Adenovirus, Stafilococo aureus, Stafilococo coagulasa negativo, Escherichia coli y Cándida (45).

Tales infecciones obligaron a la prolongación de la internación y complicaron la condición de los niños, a tal punto que requirieron traslado a las unidades de terapia intensiva y algunos reingresaron por la misma causa. En ambos estudios se reportaron casos de muerte como consecuencia de la IACS (44-45).

Al respecto, en la actualidad López et al., plantean algunas estrategias que ayudan a minimizar estos eventos adversos como: un sistema de vigilancia activo que permita su detección a tiempo, instaurando una terapéutica antimicrobiana adecuada y así disminuir la morbimortalidad de los pacientes y evitar costos al sistema de salud. Además, sugieren implementar 
medidas rutinarias de cuidado para catéter venoso periférico, que es uno de los focos para adquirir IACS (45).

Otra recomendación importante, es contar con habitaciones individuales para los niños hospitalizados con condiciones especiales y con personal entrenado en el manejo de dispositivos (accesos vasculares, catéteres de alimentación enteral y gastrostomía, entre otros) y reforzar de manera continua las medidas para la prevención tales infecciones (44).

\section{Flebitis}

De las diferentes vías que se utilizan para administración de tratamiento farmacológico a los niños, la intravenosa es la principal opción, pues a través de ella se puede, además de dar medicamentos, reponer líquidos, electrolitos, macronutrientes y micronutrientes, dados los procesos patológicos frecuentes en esta etapa del ciclo vital: infecciones, quemaduras, procedimientos quirúrgicos, deshidratación y desnutrición (46-47).

El tener un acceso intravenoso incrementa el riesgo de flebitis, que se describe como la inflamación del vaso sanguíneo y, según su naturaleza, se clasifica en: química, cuando el pH o la osmolaridad de la solución lesiona el endotelio del vaso sanguíneo; mecánica, que puede ser consecuencia del trauma generado por el calibre o inadecuada manipulación del catéter; e infecciosa, que se relaciona con la contaminación de la solución administrada, la mala asepsia del sitio de inserción o la manipulación inadecuada del catéter con agentes infecciosos. Este evento adverso se puede dar de forma local o sistémica (47).

Con la intención de identificar los factores de riesgo para flebitis relacionada con catéteres venosos periféricos en neonatos y niños, se adelantaron dos investigaciones en Brasil. La primera de Rodríguez et al., reportó este evento adverso en un $16.7 \%$ de los casos(46); mientras la segunda hecha por De Lima et al., con un $2,7 \%$, (47), razón por la cual se puede inferir que a menor edad, mayor riesgo de flebitis, por la fragilidad de la red vascular de los neonatos (46).

Dentro de las factores implicados en el fracaso de la punción y aparición de flebitis, se encuentran las características de los fármacos, las soluciones, tipos de dispositivos (46) y el uso de catéter periférico por más de cinco días (47). También los antecedentes de la flebitis, que incrementan en un $40 \%$ el riesgo (47). Se sigue documentando todo lo relacionado con los antibióticos (46) y las soluciones cuya osmolaridad es superior a $350 \mathrm{mOs} / \mathrm{L}$ y $\mathrm{pH}$ menor de 5 o mayor a 9, como los más comprometidos en la flebitis(47).

Esto obliga a promover la seguridad de los niños sometidos a terapia intravenosa, para lo cual, el equipo de enfermería debe evaluar periódicamente el acceso venoso periférico(47), así como identificar aquellos factores de riesgo que favorecen su aparición, incentivando el análisis crítico de las situaciones propias de cada paciente en lo que se refiere a la necesidad del uso de un acceso venoso central cuando la situación lo amerite, sopesando el riesgo beneficio.

Las evidencias anteriores, permiten deducir que la Enfermería debe conocer sobre el tipo de catéter utilizado, su estabilización, la técnica y sitio más seguro de inserción, las características de la solución de infusión, el tiempo de permanencia del dispositivo, entre otros. De igual manera, se debe tener en cuenta las características inherentes al paciente, como el color de la piel, el sexo, la prematuridad y ciertas condiciones clínicas como infecciones, traumas, desnutrición y quemaduras de la mano, aplicando los protocolos y guías, imprescindibles para propiciar la canalización de una vena periférica segura (46-47). Por consiguiente, es ideal la presencia de una persona con experticia en el procedimiento, pero que además tenga un acervo teórico actualizado sobre el tema y que esté asignada en los turnos y servicios más críticos.

\section{Úlceras por presión (UPP)}

Las UPP se constituyen en un gran problema de salud porque: deterioran la calidad de vida de los pacientes y su familia, aumentan el costo social, incrementan el consumo de recursos en salud y en la actualidad tienen una connotación legal importante para el equipo de salud, pues representan una complicación del cuidado de la salud que no debe ser atribuible exclusivamente al cuidado de enfermería. Si bien es cierto, que la ausencia de UPP es considerada como un indicador del cuidado de enfermería, el abordaje en la prevención y manejo debe ser multidisciplinario (48).

Aunque las UPP son más frecuentes en adultos con edad avanzada, con enfermedades severas, compromiso neurológico y de la movilidad(49), la presencia de este evento adverso también es importante en los 
ISSN-PRINT

1794-9831

E-ISSN 2322-7028

Vol. 16 No. 1

Ene - Abr 2019

Cúcuta, Colombia niños. Al respecto, se han realizado estudios en Unidades de Cuidados Intensivos Pediátrico los cuales revelan una alta presencia de UPP como el de Aparicio et al.(22) quienes hallaron que sólo el $17.1 \%$ de los niños tenía la piel integra, el 58.6\% tenía lesiones en estadio I, el $34.4 \%$ es estadio II y el $6.8 \%$ en estadio III. Por su parte, Romero y Aguilar (27), establecieron que el 37\% de los niños tenían UPP y Schindler et al. (50). señalaron que existe una incidencia del $10.2 \%$, observando un $63.4 \%$ de los casos en estadio I, $32.2 \%$ en estadio II, $3.68 \%$ estadio III y $0.9 \%$ en estadio IV. Con respecto a los sitios anatómicos más afectados por estas lesiones, se pueden mencionar los glúteos, cuello, perineo, occipucio, sacro, hombros, frente y espalda $(22,27,50)$.

Estos mismos estudios, determinaron que los niños con mayor susceptibilidad de UPP fueron los mayores de 2 años, que estuvieron en la unidad de cuidados intensivos 4 días o más $(22,50)$, que requirieron de ventilación mecánica, ventilación no invasiva, oxigenación con membrana extracorpórea (49), o necesitaron medicamentos vasopresores, inmunosupresores y anticoagulantes (27). Se debe precisar, que los pacientes en estas condiciones presentan factores de riesgo, como limitaciones en su percepción sensorial, piel húmeda, poca movilidad y nutrición probablemente inadecuada, los cuales aumentan el peligro de lesiones cutáneas. También se debe tener en cuenta la presencia de sondas, catéteres, yesos y vendajes, entre otros $(22,27)$.

Sumado a lo anterior, actividades que previenen la aparición de UPP y que son propias del cuidado de enfermería no siempre se realizan, como el estudio de Romero, que demuestra que al $33 \%$ de los niños no se les cambiaba de posición, al $72 \%$ no se les lubricaba la piel, solo al $11 \%$ se les cambiaba la sábana más una vez al día y en el $75 \%$ de los casos no se utilizaba ningún dispositivo para su prevención (27).

Otras medidas efectivas son: examinar la piel del niño cada dos horas durante la hospitalización, valoración sistémica específica y tratar las lesiones para evitar su exacerbación. También se deben usar: cojines, almohadas desechables, lociones corporales, pañales de tejido seco, colchones de presión alterna, cuñas de espuma y apósitos especiales. Igualmente, hay que estimular la educación de los cuidadores, así como la valoración por nutricionista cuando el paciente lo requiera (22).
A la luz de lo analizado en la revisión de los artículos seleccionados, se puede inferir que los niños son un universo aparte del resto de la población de pacientes, y como tal, tienen condiciones únicas que los hacen susceptibles a eventos adversos. Dichas particularidades, por ser inherentes al proceso de crecimiento y desarrollo de los infantes no se pueden modificar. No obstante, desde el cuidado de enfermería se pueden identificar tales factores con el propósito de dinamizar estrategias de gestión ajustadas a los servicios de pediatría que propendan por encender alertas y de esta manera planear y ejecutar un cuidado seguro.

En cuanto a los factores extrínsecos implicados en los eventos adversos en pediatría, tales como la falta de experticia, conocimientos y destreza, solo se podrán mejorar desde el claustro universitario, desde donde se debe formar adecuadamente al enfermero para que comprenda a cabalidad su rol respecto a la seguridad del paciente, y así planear un cuidado seguro basado en la evidencia científica, integrándolo a la condición de enfermedad del niño.

En este sentido, el desafío para los profesionales de la enfermería está en la integración del Proceso de Atención de Enfermería a la Práctica Clínica, con el objeto de impactar en la seguridad del paciente y la calidad de los servicios de salud.

\section{Conclusiones}

Los eventos adversos a los niños en el ámbito hospitalario, se constituyen en todo un desafío para el cuidado de enfermería, dado que están implicados factores propios de la naturaleza de los infantes que los hacen vulnerables a estas situaciones y que no se pueden modificar, pues son inherentes a su crecimiento y desarrollo. Así, su fragilidad vascular los hace susceptibles a la flebitis; sus cambios corporales continuos que inciden en la farmacocinética y farmacodinamia, incrementan el riesgo a EAM; la susceptibilidad de su sistema inmune, los hace propensos a las IACS; y las condiciones de su piel, a las UPP.

No obstante, según los artículos analizados, se deduce que en la salud de los niños también juegan un papel fundamental: las situaciones propias de las intervenciones, las tecnologías y el ambiente hospitalario, como la osmolaridad de los medicamentos y pertinencia de catéteres en las flebitis; el desconocimiento en farmacología y la omisión en la aplicación del uso 
correcto en la preparación y aplicación de medicamentos, en los EAM; fallas en el cuidado de la piel cuando los niños tienen dispositivos, lo que genera las UPP; e incumplimientos en las medidas para la prevención de IACS. Se debe precisar que estos aspectos están inmersos en el cuidado de enfermería y que la mayoría son conocidos, razón por la cual se deben fomentar acciones que favorezcan la cultura de seguridad en los servicios de pediatría. Por tanto, hay que reafirmar y evaluar de manera constante los conocimientos sobre las diferentes prácticas de cuidado que conllevan a la prevención de fallas.

Un aspecto fundamental a tener en cuenta en el ámbito organizacional es que se siga promoviendo la notificación anónima, no punitiva, para fortalecer la cultura del reporte, lo que permite que se deriven planes de mejoramiento con acciones correctivas y preventivas. Es imprescindible, además detectar conductas negligentes, de impericia, omisiones y descuidos, aspectos que se pueden tratar con la educación permanente y el trabajo en equipo. En el contexto individual, es necesario que cada profesional de enfermería realice una reflexión continua sobre su actuar, de tal manera que pueda identificar fallas y equivocaciones que desencadenen en EA, así como liderar acciones de mitigación y manejo adecuado en caso de que se requiera de su intervención.

\section{Conflicto de Intereses}

los autores declaran no tener ningún conflicto de interés.

\section{Referencias Bibliográficas}

1. León Román CA. La seguridad del paciente, una responsabilidad en el acto del cuidado. Rev Cubana Enfermer [Internet]. 2006 [consultado 26 de noviembre de 2017]; 22(3):1-7. Disponible en: http:// scielo.sld.cu/pdf/enf/v22n3/enf07306.pdf

2. Arratia A. Investigación y Documentación Histórica en Enfermería. Rev Contexto Enferm. [Internet]. 2005 [consultado 2 de noviembre de 2017]; 14(4): 567-574. Disponible en: http://www.scielo.br/pdf/ tce/v14n4/a14v14n4.pdf

3. República de Colombia. Ministerio de la Protección Social. Dirección General de la Calidad del Servicio. Unidad Sectorial de Normalización. Guía Técnica: Buenas Prácticas para la Seguridad del Paciente en la Atención en Salud. Bogotá: Minsalud, 2010. [consultado 13 de enero de 2018]. Disponible en: http://www.acreditacionensalud.org.co/ea/Documents/Guia\%20Buenas\%20Practicas\%20Seguridad $\% 20 \mathrm{del} \% 20$ Paciente.pdf

4. Navas J, Alvarado P, González JL, Delgado SA, Domínguez SL, et al. Eventos adversos relacionados con el cuidado de enfermería. Rev Cubana Enferm. [Internet]. 2014 [consultado 25 de noviembre de 2017]; 30(1):19-27. Disponible en: http://www.revenfermeria.sld.cu/index.php/enf/article/ view/171/80

5. Fernández N. Los eventos adversos y la calidad de atención Estrategias para mejorar la seguridad de los pacientes pediátricos. Arch Argen Pediatr [Internet]. 2004 [consultado 14 de septiembre de 2017]; 102(5):402-410. Disponible en: http://www.scielo.org.ar/pdf/aap/v102n5/v102n5a14.pdf

6. República de Colombia. Ministerio de Salud. Seguridad del Paciente y la Atención Segura: paquetes instruccionales Guía Técnica Buenas Prácticas para la Seguridad del Paciente en la Atención en Salud. Bogotá: Minsalud, 2015. [consultado 13 de enero de 2018]. Disponible en: https://www.minsalud.gov. co/sites/rid/Lists/BibliotecaDigital/RIDE/DE/CA/Guia-buenas-practicas-seguridad-paciente.pdf

7. Organización Mundial de la Salud. Más que palabras. Marco Conceptual de la Clasificación Internacional para la Seguridad del Paciente Informe Técnico Definitivo. Enero de 2009. Washington: OMS, 2009. [consultado 13 de enero de 2018]. Disponible en: http://www.who.int/patientsafety/implementation/icps/icps_full_report_es.pdf

8. Bauer AE. Segurança do paciente: desafios para a prática e a investigação em Enfermagem. Rev Eletrônica Enferm [Internet]. 2010 [consultado 18 de marzo de 2018]; 12(3): 422. Disponible en: https:// www.fen.ufg.br/revista/v12/n3/v12n3a01.htm 
ISSN-PRINT

1794-9831

E-ISSN 2322-7028

Vol. 16 No. 1

Ene - Abr 2019

Cúcuta, Colombia

9. Roque KE, Prates EC. Adaptação dos critérios de avaliação de eventos adversos a medicamentos para uso em um hospital público no Estado do Rio de Janeiro. Rev Bras Epidemiol [Internet]. 2010 [consultado 14 de septiembre de 2017]; 13(4):607-619. Disponible en: https://www.scielosp.org/article/ssm/ content/raw/?resource_ssm_path=/media/assets/rbepid/v13n4/06.pdf

10. Institute of Medicine US. Committee on Quality of Health Care in America. To Err Is Human [Libro en Internet] Washington: National Academies Press, 2000. [consultado 9 de agosto de 2017]. Disponible en: https://www.ncbi.nlm.nih.gov/books/NBK225182/

11. Schatkoski AM, Wegner W, Algeri S, Pedro ENR. Safety and protection for hospitalized children: literature review. Rev Lat Am Enfermagem [Internet]. 2009 [consultado 13 de noviembre de 2017]; 17(3): 410-6. Disponible en: http://www.scielo.br/scielo.php?script=sci arttext\&pid=S0104$11692009000300020 \& \operatorname{lng}=$ en\&tlng=en

12. República de Colombia. Ministerio de la Protección Social. Lineamientos para la implementación de la Política de Seguridad del Paciente. Bogotá: Buenos y creativos, 2008. [consultado 12 de enero de 2018]. Disponible en: https://minsalud.gov.co/Normatividad_Nuevo/Resoluci\%C3\%B2n\%20 0112\%20de\%202012\%20-\%20Documentos\%20de\%20apoyo\%202.pdf

13. Allué N, Chiarello P, Bernal E, Castells X, Giraldo P, et al. Impacto económico de los eventos adversos en los hospitales españoles a partir del Conjunto Mínimo Básico de Datos. Gac Sanit [Internet] 2014 [consultado 22 de septiembre de 2017];28(1): 48-54. Disponible en: http://dx.doi.org/10.1016/j. gaceta.2013.06.004

14. Gutiérrez LM, Torres A, Soria M, Padrón A, Ramírez ME. Costs of serious adverse events in a community teaching hospital, in Mexico. Cirugía y Cirujanos [Internet]. 2015 [consultado 21 de septiembre de 2017];83(3): 210-16. Disponible en: https://www.sciencedirect.com/science/article/pii/ $\underline{\mathrm{S} 2444050715000790}$

15. Palacios A, Bareño J. Factores asociados a eventos adversos en pacientes hospitalizados en una entidad de salud en Colombia. CES Med [Internet]. 2012 [consultado 22 de septiembre de 2017];26(1): 19-28. Disponible en: http://www.redalyc.org/html/2611/261123426003/

16. Ferreira A, Fort Z, Chiminelli V. Eventos adversos en salud y cuidados de enfermería: la seguridad del paciente desde la experiencia del profesional. Texto Context Enferm [Internet]. 2015 [consultado 25 de septiembre de 2017]; 24(2):310-15. Disponible en: http://www.redalyc.org/articulo. oa? id $=71442215003$

17. Barrionuevo L, Esandi ME. Epidemiología de eventos adversos en el servicio de neonatología de un hospital público regional en la Argentina. Arch Argent Pediatr [Internet]. 2010 [consultado 7 de octubre de 2017]; 108(4):303-10. Disponible en: http://www.scielo.org.ar/pdf/aap/v108n4/v108n4a03.pdf

18. Tolieng V, Prasirtsak B, Thongchul N, Tanasupawat S. Identification and lactic acid production of bacteria isolated from soils and tree barks. Malays J Microbiol [Internet]. 2017 [consultado 23 de septiembre de 2017]; 13(2):15-30. Disponible en: http://science.buu.ac.th/ojs246/index.php/sci/article/ view/1508/1752

19. Mendes W, Pavão AL, Martins M, De Oliveira M, Travassos C. Características de eventos adversos evitáveis em hospitais do Rio de Janeiro. Rev Assoc Med Bras. [Internet]. 2013 [consultado 20 de septiembre de 2017];59(5): 421-28. Disponible en: https://www.arca.fiocruz.br/bitstream/icict/9638/2/ Caracter\%C3\%ADsticas\%20de\%20eventos\%20adversos\%20evit\%C3\%A1veis\%20em\%20hospitais\%20do\%20Rio\%20de\%20Janeiro.pdf

20. Achury D, Rodríguez S, Díaz JC, Cavallo E, Zarate R, et al. Estudio de eventos adversos, factores y periodicidad en pacientes hospitalizados en unidades de cuidado intensivo. Enferm Glob. 2016; 15(2):324-40.

21. Gómez AI, Espinosa AF. Ethical Dilemmas Facing the Patient's. Aquichan [Internet]. 2006 [consultado 23 de septiembre de 2017]; 6(6):54-67. Disponible en: http://aquichan.unisabana.edu.co/index. 
php/aquichan/article/view/80/165

22. Aparicio G, Ponce G, Carmona B. Cuidados a la piel del niño y factores de riesgo para desarrollar úlceras por presión. Enfermería Universitaria [Internet]. 2010 [consultado 14 de septiembre de 2017]; 7(3):7-15. Disponible en: http://www.revistas.unam.mx/index.php/reu/article/view/25002/23512

23. Villarreal E, Lozano W, Mendoza S, Montenegro N, Olarte. Eventos adversos derivados del cuidado brindado en la Unidad de Cuidados Intensivos Adultos del Hospital Universidad del Norte. Barranquilla (Colombia). Salud Uninorte [Internet]. 2014 [consultado 13 de septiembre de 2017]; 30(3):38191. Disponible en: http://www.redalyc.org/pdf/817/81737153012.pdf

24. Camargo F, Parra D, Rey R. Eventos adversos derivados del cuidado de enfermería: flebitis, úlceras por presión y caídas. Enferm Glob. [Internet]. 2012 [consultado 18 de noviembre de 2018]; 28(28):159-69. Disponible en: http://revistas.um.es/eglobal/article/view/150551/139861

25. De Freitas M, de Goes MA, de Fátima A. Nursing Diagnosis Risk for falls: prevalence and clinical profile of hospitalized patients. Rev Latino-Am Enfermagen [Internet]. 2014 [Consultado 14 de septiembre de 2017]; 22(2):262-8. Disponible en: http://www.scielo.br/pdf/rlae/v22n2/0104-1169rlae-22-02-00262.pdf

26. Olvera S, Hernández A, Arroyo S, Nava MG, Zapien M, et al. Factores Relacionados con la resencia de Caídas en pacientes hospitalizados. Rev Invest Clin [Internet]. 2013 [consultado 2 de noviembre de 2017]; 65(1):88-93. Disponible en: http://www.medigraphic.com/pdfs/revinvcli/nn-2013/nn131j.pdf

27. Romero G, Aguilar E. Factores de riesgo para úlceras por presión en pacientes pediátricos hospitalizados en la Unidad de Cuidados Intensivos. Enferm Inst Mex Seguro Soc. [Internet]. 2017 [Consultado 12 de septiembre de 2017]; 25(2):111-8. Disponible en: http://www.medigraphic.com/pdfs/enfermeriaimss/eim-2017/eim172f.pdf

28. Queiroz AL, Queiroz E, Weber J, Tanferri P. Reacciones adversas: indicadores de resultados según la percepción de las enfermeras de un hospital centinela. Enfermería Glob [Internet]. 2012 [Consultado 15 de septiembre de 2017]; 11(27):186-97. Disponible en: http://revistas.um.es/eglobal/article/ view/155151

29. Bagheri M, Esmaeili R, Tajari M. Intravenous Medication Administration Errors and their Causes in Cardiac Critical Care Units in Iran. Mater Socio Medica [Internet]. 2015 [Consultado 22 de septiembre de 2017]; 27(6):442-6. Disponible en: http://www.scopemed.org/fulltextpdf.php?mno=209963

30. Pereira J, Bauer A, Queiroz AL, Gomes MR, Alves I. Eventos adversos identificados nos relatórios de enfermagem em uma clínica pediátrica. Cienc y enfermería [Internet]. 2014 [Consultado 30 de agosto de 2017]; 20(2):53-63. Disponible en: http://www.scielo.cl/scielo.php?script=sci_arttext\&pid=S071795532014000200006\&lng=en\&nrm=iso\&tlng=en

31. Urrútia G, Bonfill X. Declaración PRISMA: una propuesta para mejorar la publicación de revisiones sistemáticas y metaanálisis. Med Clin (Barc). [Internet]. 2010 [Consultado 17 de noviembre de 2017]; 135(11):507-11. Disponible en: http://www.laalamedilla.org/Investigacion/Recursos/PRISMA\%20 Spanish\%20Sept\%202010.pdf

32. Cascaes F, Valdivia BA, da Rosa R, Barbosa PJ, da Silva R. Escalas y listas de evaluación de la calidad de estudios científicos. Rev Cuba Inf en Ciencias la Salud [Internet]. 2013 [Consultado 17 de noviembre de 2017]; 24(3):295-312. Disponible: http://scielo.sld.cu/scielo.php?script=sci_arttext\&pid=S230721132013000300007\&lng=es\&nrm=iso\&tlng=es

33. Von Elm, Altman DG, Egger M, Pocock SJ, Gotzsche PC, Vandenbroucke JP. Declaración de la Iniciativa STROBE (Strengthening the Reporting of Observational studies in Epidemiology): directrices para la comunicación de estudios observacionales. Gac Sanit [Internet]. 2008 [Consultado 17 de noviembre de 2017]; 22(2):144-50. Disponible en: https://www.strobe-statement.org/fileadmin/ Strobe/uploads/translations/STROBE_short_Spanish.pdf

34. Vandenbroucke JP, Von Elm E, Altman DG, Gøtzsche PC, Mulrow CD, Pocock SJ, et al. Mejorar la 
ISSN-PRINT

1794-9831

E-ISSN 2322-7028

Vol. 16 No. 1

Ene - Abr 2019

Cúcuta, Colombia

comunicación de estudios observacionales en epidemiología (STROBE): explicación y elaboración. Gac Sanit. [Internet]. 2008 [Consultado 17 de noviembre de 2017]; 23(2):1-28. Disponible en: https:// www.strobe-statement.org/fileadmin/Strobe/uploads/translations/STROBE-Exp-SPANISH.pdf

35. Tong A, Sainsbury P, Craig J. Consolidated criterio for reporting qualitative research (COREQ): a 32- item checklist for interviews and focus group. Int J Qual Heal Care. [Internet]. 2007 [Consultado 17 de noviembre de 2017]; 19(6):349-57. Disponible en: https://academic.oup.com/intqhe/article/19/6/349/1791966

36. República de Colombia. Ministerio de Salud. Resolución No 008430 de 1993, octubre 4, por la cual se establecen las normas científicas, técnicas y administrativas para la investigación en salud [Internet]. Santa Fe de Bogotá: El Ministerio; 1993 [consultado 5 de julio de 2017]. Disponible en: https://www. minsalud.gov.co/sites/rid/Lists/BibliotecaDigital/RIDE/DE/DIJ/RESOLUCION-8430-DE-1993.PDF

37. Requena J, Miralles JJ, Mollar J, Aranaz JM. Seguridad clínica de los pacientes durante la hospitalización en pediatría. Rev Calid Asist. [Internet]. 2011 [Consultado 22 de noviembre de 2017];26(6): 353-8. Disponible en: http://www.elsevier.es/es-revista-revista-calidad-asistencial-256-articulo-seguridad-clinica-los-pacientes-durante-S1134282X11001588? redirectNew=true

38. Gutiérrez S, Mogni A, Berón A, Iramain R. Errores de medicación en niños hospitalizados. Arch Pediatr Urug. [Internet]. 2011 [Consultado 23 de septiembre de 2017]; 82(3):133-40. Disponible en: http:// www.scielo.edu.uy/pdf/adp/v82n3/v82n3a02.pdf

39. Riquelme G, Antognoni A. Descripción de eventos adversos en un hospital pediátrico de la ciudad de Santiago, Chile. Enferm Glob. [Internet]. 2013 [Consultado 23 de septiembre de 2017];12(1): 262-73. Disponible: http://scielo.isciii.es/pdf/eg/v12n29/administracion4.pdf

40. Wegner W, Rubim E. La seguridad del paciente en las circunstancias de cuidado. Rev Latino-Am Enferm. [Internet]. 2012 [consultado 18 de agosto de 2017]; 20(3):1-8. Disponible en: http://www.scielo. br/pdf/rlae/v20n3/es_a02v20n3.pdf

41. Esqué MT, Moretones MG, Rodríguez JM, Sánchez E, Izco M, de Lamo M, et al. Medication errors in a neonatal unit: One of the main adverse events. An Pediatría [Internet]. 2016 [consultado 27 de febrero de 2018]; 84(4):211-7. Disponible en: https://ac.els-cdn.com/S2341287916000235/1-s2.0S2341287916000235-main.pdf? tid=84c1ad25-7549-44d6-a550-cde3ace3b286\&acdnat $=152226460$ $4 \_785 \mathrm{a} 1 \mathrm{e} 6 \mathrm{aabcf} 972 \mathrm{e} 9 \mathrm{f} 91 \mathrm{~b} 3 \mathrm{bf} 2848 \mathrm{ef} 10$

42. Tayman C, Rayyan M, Allegaert K. Neonatal pharmacology: extensive interindividual variability despite limited size. J Pediatr Pharmacol Ther [Internet]. 2011 [consultado 23 de agosto de 2017]; 16(3):170-84. Disponible en: http://www.pubmedcentral.nih.gov/articlerender.fcgi?artid=3292528\&t ool $=$ pmcentrez\&rendertype $=$ abstract

43. Organización Mundial de la Salud. Carga mundial de infecciones asociadas a la atención sanitaria [Internet]. OMS, 2013 [consultada 27 de febrero de 2018]. Disponible en: http://www.who.int/gpsc/ country_work/burden_hcai/es/

44. Flores JC, Riquelme P, Cerda J, Carrillo D, Matus MS, Araya G, et al. Mayor riesgo de infecciones asociadas a atención en salud en niños con necesidades especiales hospitalizados. Rev Chil infectología [Internet]. 2014 [consultada 20 de noviembre de 2017]; 31(3):287-92. Disponible en: http:// www.scielo.cl/scielo.php?script=sci_arttext\&pid=S0716-10182014000300006\&lng=en\&nrm=iso\&tl ng=en

45. López D, Aurenty L, Rosales T, Quines M, Guerra J, Goncalves M, et al. Infecciones asociadas a cuidados de la salud en pacientes pediátricos con cáncer. Rev Venez Oncol. [Internet]. 2015 [consultada 25 de enero de 2018]; 27(3):156-64. Disponible en: http://www.imbiomed.com.mx/1/1/articulos. php? $m e t h o d=$ showDetail\&id_articulo $=105826 \&$ id_seccion $=1549 \&$ id_ejemplar $=10282 \&$ id_revis$\underline{\text { ta }=100}$

46. Rodríguez AC, Gamarra CJ, de Oliveira JC, Machado AF, Rodrigues E, et al. Assessment of phlebitis, 
infiltration and extravasation events in neonates submitted to intravenous therapy. Esc Anna Nery [Internet]. 2011 [consultado 30 de enero de 2018]; 15(3):472-9. Disponible en: http://www.scielo.br/ scielo.php?script $=$ sci_arttext\&pid $=$ S1414-81452011000300005\&lng=en\&tlng $=$ en

47. De Lima AK, Machado M, Martins AM, Goncalves M. Phlebitis associated with peripheral intravenous catheters in children: study of predisposing factors. Esc Anna Nery - Rev Enferm [Internet]. 2014 [consultado 30 de noviembre de 2017]; 18(2):220-6. Disponible en: http://www.gnresearch.org/ doi/10.5935/1414-8145.20140032

48. República de Colombia. Ministerio de Salud. Prevención Úlceras Por Presión: paquetes instruccionales Guía Técnica Buenas Prácticas para la Seguridad del Paciente en la Atención en Salud. Bogotá: Minsalud, 2015. [consultado 13 de enero de 2018]. Disponible en: https://www.minsalud.gov.co/sites/ rid/Lists/BibliotecaDigital/RIDE/DE/CA/prevenir-ulceras-por-presion.pdf

49. National Pressure Ulcer Advisory Pane, European Pressure Ulcer Advisory Panel and Pan Pacific Pressure Injury Alliance. Prevention and Treatment of Pressure Ulcers: Quick Reference Guide [Guía en Internet]. Australia, 2014. [consultado 27 de febrero de 2018]. Disponible en: https://www.npuap. org/wp-content/uploads/2014/08/Updated-10-16-14-Quick-Reference-Guide-DIGITAL-NPUAPEPUAP-PPPIA-160ct2014.pdf

50. Schindler CA, Mikhailov TA, Kuhn EM, Christopher J, Conway P, Ridling D, et al. Protecting fragile skin: Nursing interventions to decrease development of pressure ulcers in pediatric intensive care. Am J Crit Care [Internet]. 2011 [consultado 27 de febrero de 2018]; 20(1): 26-35. Disponible en: http:// www.ncbi.nlm.nih.gov/pubmed/21196569 